Case Report

\title{
Severe Pansinusitis Due to Raoultella Ornithinolytica
}

\author{
Mitchell R. Gore \\ Department of Otolaryngology, \\ State University of New York-Upstate Medical University, Syracuse, NY, USA
}

Article history

Received: 23-09-2017

Revised: 05-12-2017

Accepted: 20-12-2017

Tel: +13154923741

Fax: +1 3154925791

Email: mgoremdphd@gmail.com

\begin{abstract}
Raoultella ornithinolytica is a gram-negative aerobic bacterium typically found in plants, soil and water. Reports of Raoultella ornithinolytica causing infections in humans, especially in the head and neck, are rare. Herein we report a case of a woman with severe septal deviation, chronic headaches and facial pain who was revealed to have Raoultella ornithinolytica pansinusitis incidentally noted during an endoscopic septoplasty. Raoultella ornithinolytica was isolated from cultures of the diffuse purulent drainage found in her maxillary and ethmoid sinuses. This case indicates that while a rare cause of head and neck infection in humans, Raoultella ornithinolytica can be found as the causative pathogen in severe sinusitis.
\end{abstract}

Keywords: Raoultella ornithinolytica, Sinusitis, Infection

\section{Introduction}

Raoultella ornithinolytica is a member of a genus of Gram-negative, oxidase-negative, aerobic, nonmotile, capsulated, facultatively anaerobic rods closely related to the Klebsiella genus in the Enterobacteriaceae family. The genus is named after noted French bacteriologist Didier Raoult (Drancourt et al., 2001). Raoultella bacteria, including Raoultella ornithinolytica, are rare causes of infections in immunocompetent humans (Singh et al., 2017; Casarsa et al., 2017). Raoultella ornithinolytica may also be found in ticks, fish and in watery environments (Morais et al., 2009). Only limited case reports are available showing Raoultella species causing head and neck infections or sepsis. We report an unusual case of severe pansinusitis due to communityacquired Raoultella ornithinolytica that resolved with endoscopic sinus surgery and oral antibiotics.

\section{Case Report}

A 56-year-old woman was referred to the author's outpatient Otolaryngology practice for two to three months of nasal congestion, facial pressure and facial pain. Her past medical history was significant only for hypertension treated with hydrochlorothiazide and was negative for diabetes or autoimmune diseases. Of note the patient noted a recent history of repeatedly cleaning her elderly parents' home, which was apparently in a state of poor hygiene. She had been previously treated with a 10day course of amoxicillin and 2 weeks of intranasal fluticasone spray. She was a febrile and in no acute distress. Head and neck and cranial nerve examinations were normal. Nasal endoscopy revealed severe bilateral inferior turbinate hypertrophy and severe septal deviation causing bilateral nasal obstruction. The inferior turbinate hypertrophy and septal deviation were so significant that the middle meatus could not be visualized. Given the patient's complaints she was counseled on the risks, benefits and alternatives of endoscopic septoplasty and sub mucosal reduction of the inferior turbinates and the patient elected to undergo septoplasty and turbinate reduction. At the time of her surgery, endoscopic septoplasty and submucosal inferior turbinate reduction were performed in the standard fashion (Chandra, 2017). Once the septoplasty and turbinate reduction were completed bilaterally, the middle meatus and maxillary and ethmoid drainage pathways were easily visible. Copious white, opaque purulent fluid was noted draining from the maxillary and ethmoid sinuses bilaterally. The surgery was paused and the patient's family was counseled on the findings and intraoperative consent was obtained from the family to proceed with endoscopic sinus surgery to clear the copious purulent sinus secretions. Bilateral maxillary, ethmoid and sphenoid sinusotomies were made endoscopically, revealing white purulent fluid filling the bilateral maxillary, ethmoid and sphenoid sinuses. Figure 1 shows an endoscopic view of the left nasal cavity using a $0^{\circ} \mathrm{C}$ endoscope showing the purulent fluid draining from the left maxillary sinus prior to the antrostomy. This purulent drainage was cultured for anaerobes and aerobes bilaterally and the sinuses were irrigated with $250 \mathrm{~mL}$ per side of sterile saline irrigation once the sinuses were opened bilaterally. 


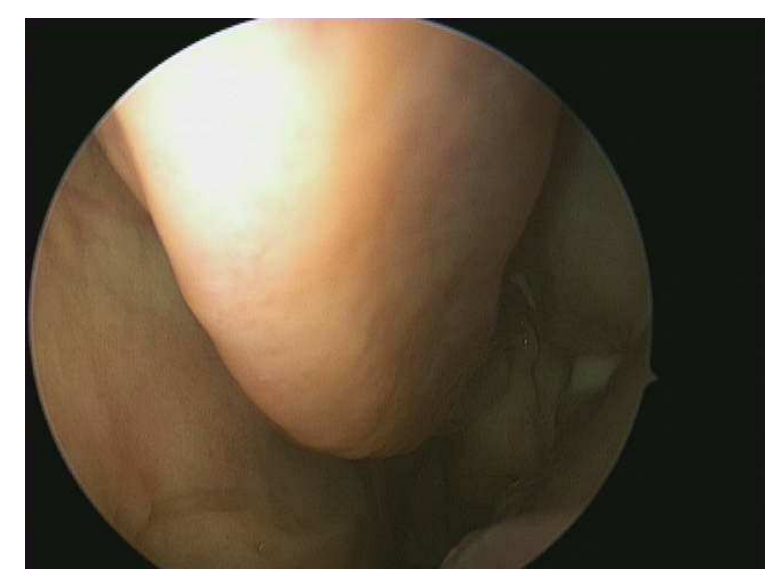

Fig. 1: Endoscopic view of the left nasal cavity showing purulent secretions draining from the left maxillary sinus

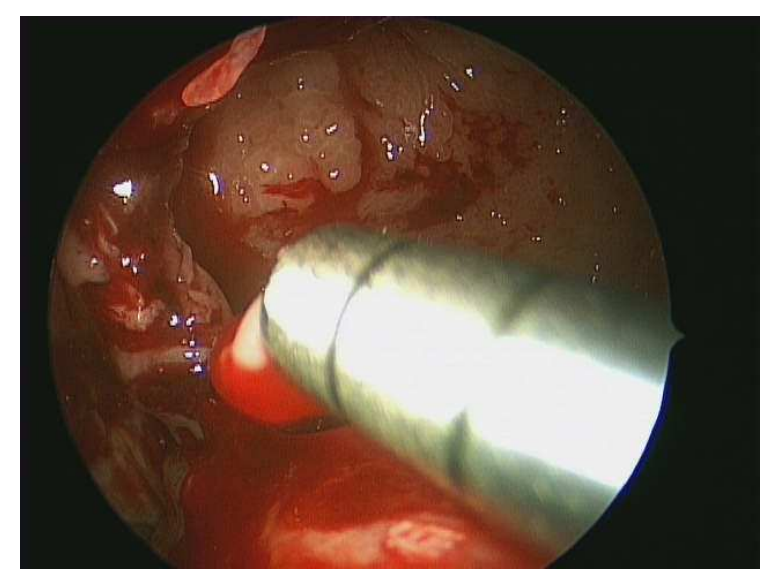

Fig. 2: Endoscopic view of the left maxillary sinus antrostomy. Note the purulent secretions being suctioned from the sinus and the inflamed, granular appearance of the sinus mucosa

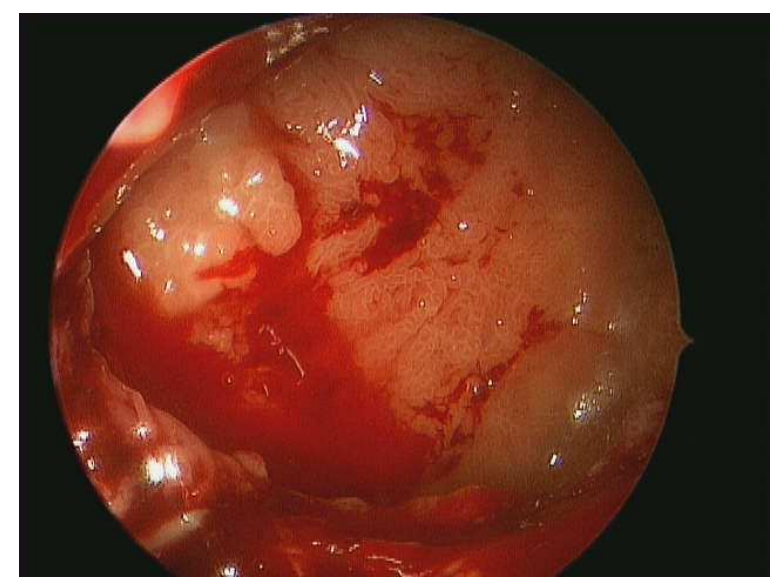

Fig. 3: Endoscopic view of the left maxillary antrostomy using an angled 45-degree endoscope after suctioning and irrigation of the sinus. Note the chronically inflamed, granular appearance of the maxillary sinus mucosa
Figure 2 shows an endoscopic view of the left maxillary antrostomy with suctioning of the purulent fluid from the sinus and Fig. 3 shows an endoscopic view with an angled $45^{\circ} \mathrm{C}$ endoscope of the left maxillary sinus after opening of the sinus, illustrating the chronically inflamed, granular appearance of the left maxillary sinus mucosa. The patient was discharged from the outpatient surgical center after her surgery in good condition on oral doxycycline twice per day $\mathrm{x} 7$ days and seen at one week post-operatively for endoscopic examination and debridement. Doxycycline was chosen as the empirical post-operative antibiotic given its broad coverage of gram-positive bacteria and its coverage of methicillinresistant Staphylococcus aureus often found in sinus cultures in the author's region. The maxillary, ethmoid and sphenoid cavities were noted to be widely patent and healing normally, with no residual purulent fluid noted in the paranasal sinuses. The surgical specimen showed bilateral chronically inflamed but benign respiratory typesinus mucosa consistent with chronic sinusitis. Her intraoperative sinus culture showed Raoultella ornithinolytica sensitive to levofloxacin, ciprofloxacin, amoxicillin-clavulanate, aztreonam, cefepime, cefotaxime, cefoxitin, ceftazidime, gentamicin, imipenem and trimethoprim-sulfamethoxazole and resistant to ampicillin. Given the culture results the patient was switched to oral levofloxacin x 10 days and seen back at 4 weeks post-operatively for repeat endoscopy. Sinonasal endoscopy revealed widely patent maxillary, ethmoid and sphenoid sinusotomies, with completely healed sinus mucosa and no residual purulence and a midline septum with nicely reduced inferior turbinates. The patient's facial pain and pressure and nasal obstruction symptoms were completely resolved. With periodic follow up and endoscopic examination of the sinuses the patient had no return of her symptoms or sinusitis for two years at the time of this report.

\section{Discussion}

Raoultella ornithinolytica is a Gram-negative anaerobic bacillus belonging to the Enterobacteriaceae family and closely related to Klebsiella. It is typically found in plants, soil, or aquatic environs and may also be found in ticks and fish. Human infection is rare, but there have been several reports of Raoultella infection in humans in the literature. Singh et al. (2017) reported a case of an elderly female patient in India with right sided otitis, pharyngitis, maxillary sinusitis and right recurrent laryngeal nerve and facial nerve palsy due to Raoultella ornithinolytica isolated on throat culture. The related bacterium Raoultella planticola has recently been reported to have caused a knee joint septic arthritis (Bonnet et al., 2017) and severe sepsis (Casarsa et al., 2017) in separate cases. Raoultella bacteria have been 
isolated in human sputum, stools, wounds and urine and have also been reported to cause biliary sepsis (Teo et al., 2012). Many reports of Raoultella systemic infection appear to be associated with a history of a recent surgical procedure or some sort of immunocompromise. Luongo reported a recent case of brain abscess caused by Raoultella ornithinolytica (Luongo, 2017). Interestingly, the patient was also noted to have sinusitis on MRI, but nasal culture showed only Candida albicans. The patient was treated with frontobasal craniotomy to drain, culture and lavage the frontobasal brain abscess and cultures from the brain abscess grew Raoultella ornithinolytica. The patient recovered with intravenous ceftriaxone and metronidazole. Fortunately, most Raoultella isolates are sensitive to several classes of antibiotics and prompt antibiotic therapy appears to resolve even systemic infections. The mainstay of treatment appears to be surgery when indicated for sinusitis or joint or intraabdominal abscesses, with empiric followed by culture-directed antibiotics, or primary antibiotics in cases with systemic infection in the absence of abscess or sinusitis. As seen in the present case, symptoms seen with Raoultella sinusitis can include facial pain, nasal congestion and sinus pressure, similar to symptoms of sinusitis seen with more common etiologic bacteria such as Streptococci or Staphylococci. Similar to more common etiologic bacteria, Raoultella sinusitis can lead to orbital or intracranial infections such as orbital cellulitis or brain abscess. The data on Raoultella sinusitis is limited, but culture directed antibiotics and thorough endoscopic sinus surgery with lavage and suctioning of the infected sinuses when refractory to antibiotics appears to be the mainstay of treatment, similar to sinusitis caused by Streptococci or Staphylococci. As the symptoms of Raoultella sinusitis can be similar to those seen with more common bacteria, Raoultella bacteria should be kept in the differential of possible organisms causing chronic sinusitis. Patients with particularly unhygienic living conditions or exposure to environments known to contain Raoultella ornithinolytica such as those containing sewage or human waste should heighten the suspicion for Raoultella as a possible causative agent in chronic sinusitis.

Sedaghat (2017), Barshak and Durand (2017), van Zele et al. (2010) and Head et al. (2016) provided excellent reviews of empirical antimicrobial selection for the management of sinusitis. Macrolides or penicillin derivatives such as amoxicillin are typically recommended for first line treatment of acute or chronic sinusitis, usually for 7 to 10 days. Patients with unresolved or prolonged symptoms can be treated with fluoroquinolones such as levofloxacin, tetracyclines such as doxycycline, or beta-lactamase resistant antibiotics such as amoxicillin-clavulanate, often for 10 to 21 days.
As noted above, Raoultella species are typically sensitive to fluoroquinolones,

In the case of the patient described in the present report, the exposure to poor hygiene in her parents' dwelling appears to have been the inciting event, with her severe nasal septal deviation contributing to poor drainage of the paranasal sinuses. As Raoultella have been found in urine and feces, as well as aquatic environs such as sewage and non-potable water, this likely caused the introduction of the Raoultella bacteria into the sinuses, with the septal deviation and sinus outflow tract obstruction and edema allowing the bacteria to opacify the sinuses. Similarly to more common causes of chronic sinusitis, introduction of bacteria found in the environment or in sick contacts are introduced into the sinuses and mucosal inflammation, sinus outflow obstruction and edema allows the bacteria to flourish, causing sinusitis. Concomitant edema from allergic rhinitis or sinonasal polyposis can contribute to the sinus inflammation and bacterial sinusitis.

Raoultella ornithinolytica can be identified on a Gram stain as a Gram-negative anaerobic bacillus closely related to Klebsiella, but may be difficult to recognize due to its rarity as a human pathogen. Alves et al. (2007) noted that Raoultella ornithinolytica can appear similar to Klebsiella, but culture and biochemical tests will typically show fermentation of lactose and acid production on MacConkey agar and Raoultella will typically be oxidase- and Voges-Proskauer-negative. Additionally, Raoultella should not produce $\mathrm{H}_{2} \mathrm{~S}$ and will typically not show arginine or phenylalanine utilization. In the Alves study Raoultella fermented glucose, utilized citrate, lysine, malonate and l-sorbose and were urease- and methyl red-positive and were nonmotile, positive for indole production, histamine assimilation and growth at $10^{\circ} \mathrm{C}$. Raoultella isolates were not noted to utilize ornithine or d-melezitose. Gene sequencing (Drancourt et al., 2001) can also be used to confirm the diagnosis of Raoultella ornithinolytica if Gram stain or biochemical identification is uncertain.

\section{Conclusion}

Raoultella ornithinolytica is a rare cause of sinusitis but should be in the differential of possible causative organisms in sinus infections, as well as head and neck, joint and intraabdominal infections. Raoultella ornithinolytica and related Raoultella bacteria in the same genus may cause serious infections even in immunocompetent patients. Prompt antibiotic therapy, combined with surgery when indicated, typically results in a favorable outcome.

\section{Ethics}

This article is original and contains unpublished material. 


\section{References}

Alves, M.S., L.W. Riley and B.M. Moreira, 2007. A case of severe pancreatitis complicated by Raoultella planticola infection. J. Med. Microbiol., 56: 696-698. DOI: 10.1099/jmm.0.46889-0

Barshak, M.B. and M.L. Durand, 2017. The role of infection and antibiotics in chronic rhinosinusitis. Laryngoscope Invest. Otolaryngol., 23: 36-42. DOI: 10.1002/lio2.61

Bonnet, E., F. Julia, G. Giordano and J. Lourtet-Hascoet, 2017. Joint infection due to Raoultella planticola: first report. Infection, 45: 703-704. DOI: $10.1007 / \mathrm{s} 15010-017-1006-3$

Casarsa, C., F. Mearelli and G. Biolo, 2017. Severe sepsis due to Raoultella planticola. Am. J. Infect. Dis., 13: 1-2. DOI: 10.3844/ajidsp.2017.1.2

Chandra, RK. 2017. Endoscopic septoplasty: "How I do it". Am. J. Rhinol. Allergy, 31: 276-277. DOI: 10.2500/ajra.2017.31.4436

Drancourt, M., C. Bollet, A. Carta and P. Rousselier, 2001. Phylogenetic analyses of Klebsiella species delineate Klebsiella and Raoultella gen. nov., with description of Raoultella ornithinolytica comb. nov., Raoultella terrigena comb. nov. and Raoultella planticola comb. nov. Int. J. Syst. Evol. Microbiol., 51: 925-32. DOI: 10.1099/00207713-51-3-925

Head, K., L.Y. Chong, P. Piromchai, C. Philpott and A.G.M. Schilder et al., 2016. Systemic and topical antibiotics for chronic rhinosinusitis. Cochrane Database Syst. Rev., 4: CD011994-CD011994. DOI: 10.1002/14651858.CD011994.pub2
Luongo, M., 2017. Rare case of cryptogenic brain abscess caused by Raoultella ornithinolityca. World J. Clin. Cases, 16: 299-302.

DOI: 10.12998/wjcc.v5.i7.299

Morais, V.P., M.T. Daporta, A.F. Bao, M.G. Campello and G.Q. Andrés, 2009. Enteric fever-like syndrome caused by Raoultella ornithinolytica (Klebsiella ornithinolytica). J. Clin. Microbiol., 47: 868-869. DOI: $10.1128 /$ JCM.01709-08

Sedaghat, A.R., 2017. Chronic rhinosinusitis. Am. Fam. Physician, 96: 500-506.

Singh, M., I. Kaur, D.K. Mundi and A. Kaur, 2017. ENT infection caused by Raoultella ornithinolytica. Niger J. Clin. Pract., 20: 914-917. DOI: 10.4103/njcp.njcp_337_16

Teo, I., J. Wild, S. Ray and D. Chadwick, 2012. A rare case of cholecystitis caused by Raoultella planticola. Case Rep. Med., 2012: 601641-601641. DOI: $10.1155 / 2012 / 601641$

van Zele, T., P. Gevaert, G. Holtappels, A. Beule and P.J. Wormald et al., 2010. Oral steroids and doxycycline: Two different approaches to treat nasal polyps. J. Allergy Clin. Immunol., 125: 1069-1076.e4. DOI: $10.1016 /$ j.jaci.2010.02.020 